\title{
Investigation of Non-Newtonian Fluid Effects during Transient Flows in a Pipeline
}

\author{
Ali Majd ${ }^{1}$ - Ahmad Ahmadi $1,{ }^{*}$ - Alireza Keramat ${ }^{2}$ \\ 1 Shahrood University, Civil Engineering Department, Iran \\ 2 Jundi Shapur University of Technology, Engineering Department, Iran
}

A sudden change in the flow rate brings about significant pressure oscillations in the piping system, known as water hammer (fluid hammer). Unsteady flow of a non-Newtonian fluid due to instantaneous valve closure is studied. Power law and Cross models are used to simulate nonNewtonian effects. Firstly, the appropriate governing equations are derived and then, they are solved by a numerical approach. A fourth-order Runge-Kutta scheme is used for the time integration, and the central difference scheme is employed for the spatial derivatives discretization. To verify the proposed mathematical model and numerical solution, a comparison with corresponding experimental results from the literature are made. The results reveal a remarkable deviation in pressure history and velocity profile with respect to the water hammer in Newtonian fluids. The significance of the non-Newtonian fluid behaviour is manifested in terms of the drag reduction and line packing effect observed in the pressure history results. A detailed discussion regarding the fluid viscosity and its shear-stress diagrams are also included.

Keywords: transient pipe flow, generalized Newtonian fluid, shear thinning fluids

Highlights

- Unsteady flow equations developed for non-Newtonian fluids.

- $\quad$ The numerical method employed for non-Newtonian transient fluid flow equations.

- Flow characteristics in a pipe section have been considered in detailed.

- Significant changes observed in drag reduction and line packing effect.

\section{INTRODUCTION}

Transient flows associated with the water hammer phenomenon are commonly encountered in both natural and engineering systems, such as hydraulic systems, oil transportation systems, and human arterial network. Sudden changes in pressurized pipe flow conditions caused by valve closure, pump operation, etc. are routine events. The excitations arising from these transient events can cause significant pressures leading to devastating forces [1].

The flow of non-Newtonian fluids and slurries in pipes occurs in a wide range of practical applications in the processing industries and many natural systems. If the fluid has a significant yield stress, or if its effective viscosity is high, industrially relevant flow rates may occur in the laminar flow regime.

The fluids under consideration in this study are shear-thinning non-Newtonian, whose rheology is described by a generalized Newtonian fluid (GNF) model, i.e. the dependency of isotropic viscosity on flow properties [2] and [3]. In the specific fluids of the present work, the viscosity can be described using either the power law or Cross models. The capability of these models has been investigated by several researchers, including such as Pinho and Whitelaw [4], Toms [5] and Bird et al. [2] in experimental and numerical studies.
To conduct computations on non-Newtonian fluids, the strain rate has to be evaluated. It requires a two-dimensional analysis to provide the velocity profile of a flow cross section. The two-dimensional analysis and computations of unsteady pressure and velocity profiles during water hammer have been developed by several researchers [6] to [10]. Pezzinga proposed a quasi-two-dimensional model for the unsteady turbulent flow of a pipe network and obtained better results than 1D models [11] and [12]. Vardy and Brown [13] have had significant contributions on non-Newtonian unsteady pipe flows especially for modelling fluids with time-dependent viscosities. More recently, Wahba [14] compared shear-thinning and shear-ticking fluids in response to a water hammer event using the power law model.

Herein, unsteady pipe flow of a non-Newtonian fluid is studied. This work may be seen as a new extension to the classic water hammer model in which transients of a Newtonian fluid contained in a straight elastic pipe supported at the valve and along the pipeline with sufficient longitudinal anchors to suppress fluid-structure interaction effects is investigated. Having done this fluid hammer simulation for the power law and Cross models, several alternate works (in terms of studying the other effects [15]) on the transients of these fluids can be offered for future research. They include viscosity 
of the pipe wall, fluid-structure interaction, column separation, each of which or any of their combinations, e.g. Ahmadi and Keramat [16]; [15], Soares et al. [17], Hadj-Taïeb and Hadj-Taïeb [18] in conjunction with the present non-Newtonian behaviour can reveal new aspects of transient flow in possible systems of corresponding significance.

Pezzinga et al. analysed transients in pressurized polymeric pipes using a two-dimensional (2D) KelvinVoigt viscoelastic model [19]. Differences between the transients in viscoelastic and elastic pipes are pointed out by considering a $2 \mathrm{D}$ model. They showed that viscoelastic models precisely represent a faster decay of pressure oscillations and velocity profiles because of a time-lag between pressure oscillations and retarded circumferential strain. Brunone et al. [20] and Kim [21] considered pressure and energy dissipation and unsteady friction in laminar transient flows. They compare their numerical result with those of experiments. Meniconi et al. also studied rapidly decelerating turbulent pipe flow. They proposed a new approach to estimate energy dissipation and pressure decay [22] and [23].

In the present study, laminar transient nonNewtonian pipe flow is simulated using the power and Cross models. To this aim, the quasi-2D equations of water hammer for non-Newtonian fluids are derived and then they are solved with appropriate numerical solutions based on the finite difference method. Computational results are provided in terms of velocity, shear stress, and viscosity distribution at the flow cross section in the middle of the pipeline. The results reveal that the non-Newtonian fluid effects significantly contribute to cross-sectional flow characteristics.

\section{MATHEMATICAL MODELLING}

\subsection{Governing Equations}

The continuity equation for transient pipe flow in a cylindrical coordinate system is as follows [24]:

$$
\begin{aligned}
& \left(\frac{\partial \rho}{\partial t}+v_{r} \frac{\partial \rho}{\partial r}+\frac{v_{\theta}}{r} \frac{\partial \rho}{\partial \theta}+v_{z} \frac{\partial \rho}{\partial z}\right)+ \\
& +\rho\left(\frac{1}{r} \frac{\partial\left(r v_{r}\right)}{\partial r}+\frac{1}{r} \frac{\partial v_{\theta}}{\partial \theta}+\frac{\partial v_{z}}{\partial z}\right)=0,
\end{aligned}
$$

where $v_{r}, v_{\theta}, v_{z}$ are radial, angular and axial velocity components, $\rho$ is density and $\mathrm{t}$ is time. The momentum equation in a cylindrical coordinate in an axial direction is:

$$
\begin{aligned}
& \rho\left(\frac{\partial v_{z}}{\partial t}+v_{r} \frac{\partial v_{z}}{\partial r}+\frac{v_{\theta}}{r} \frac{\partial v_{z}}{\partial \theta}+v_{z} \frac{\partial v_{z}}{\partial z}\right)= \\
& =\rho g_{z}+\frac{\partial \tau_{r z}}{\partial r}+\frac{1}{r} \frac{\partial \tau_{\theta z}}{\partial \theta}+\frac{\partial \tau_{z z}}{\partial z}+\frac{\tau_{z r}}{r},
\end{aligned}
$$

where $\tau_{i j}(i, j=r, \theta, z)$ are stress components in the liquid in the corresponding surface and directions. To derive unsteady flow equations for a non-Newtonian fluid some assumptions and simplifications to be stated in the following are made. The flow is quasi-twodimensional. The term 'quasi' indicates that $v_{\theta}, v_{r}=0$ meaning that $v_{z}$ is the only velocity component that varies along radial and axial directions. The convective terms are neglected [25] and [26]. The nonNewtonian fluid behaviour is based on power law and Cross models. The equation of state: $\partial \rho c^{2}=\rho g \partial H$ [25] is valid, where $H$ and $c$ are pressure head and wave speed, respectively.

With the application of the assumptions above as well as some algebraic simplifications, the continuity Eq. (1) is reduced to:

$$
\frac{g}{c^{2}}\left(\frac{\partial H}{\partial t}+v_{z} \frac{\partial H}{\partial z}\right)+\frac{\partial v_{z}}{\partial z}+\frac{1}{r} \frac{\partial\left(r v_{r}\right)}{\partial r}=0
$$

Integration over the flow cross section and neglecting convective terms leads Eq. (3) to:

$$
\frac{\partial H}{\partial t}+\frac{c^{2}}{g} \frac{\partial \bar{V}}{\partial z}=0
$$

in which $\bar{V}$ is the average velocity over the flow cross section. Likewise, Eq. (2) with considering the assumptions above yields:

$$
\frac{\partial v_{z}}{\partial t}+v_{z} \frac{\partial v_{z}}{\partial z}+v_{r} \frac{\partial v_{z}}{\partial r}=-g \frac{\partial H}{\partial z}+\frac{1}{r \rho} \frac{\partial r \tau}{\partial r},
$$

The convective terms in the above equation may be neglected so that it reduces to:

$$
\frac{\partial v_{z}}{\partial t}+g \frac{\partial H}{\partial z}=\frac{1}{r \rho} \frac{\partial r \tau}{\partial r} .
$$

The shear stress term on the right-hand side of Eq. (6) represents the fluid dynamic forces and is be calculated by the constitutive rheological fluid property.

So far, no particular assumption is made for the type of fluid in governing equations; consequently, the above equations are valid for all fluid types.

\subsection{Non-Newtonian Fluid Equations}

Non-Newtonian fluids may be classified into three general classes: time independent, time dependent and 
viscoelastic fluids [3]. Among them, the first branch is investigated in transient flows of the present study.

Time independent fluids, which are placed in the inelastic fluids category, are also known as generalized Newtonian fluids (GNF). This category is similar to Newtonian fluids, but the shear stress and rate of deformation tensor are no longer a linear relation anymore. In fact, shear stresses are a nonlinear function of the rate of deformation. This nonlinear function is originated from natural features. On this basis, this category is divided into that with a yield stress and without yield stress. In the no yield stress group, there are two types: pseudo-plastics and dilatant fluids. The simulation of the former is the focus of this article.

The viscous fluid flow is defined in terms of the velocity gradient that includes the rate of deformation and spin tensor. The constitutive relation between the shear stress $\tau$ in Eq. (6) and the shear rate of the fluid is:

$$
\tau=\eta \dot{\gamma}_{y x},
$$

where $\eta$ and $\dot{\gamma}_{x y}$ are apparent viscosity and shear rate respectively. Power law, Carreau, Cross, Ellis, etc. are different models that exist in the literature for the mathematical modelling of pseudo plastics, each of which has strengths and weaknesses [3]. In this study, the power law and the Cross model are applied.

The power law is the simplest with the fewest possible parameters. It is described by the following equation:

$$
\eta=m\left(\dot{\gamma}_{y x}\right)^{n-1}
$$

where $m$ and $n$ are two empirical curve fitting parameters and are known as the fluid consistency coefficient and the flow behaviour index respectively. In this equation, if $n$ equals one and $m$ is set to $\eta_{0}$, the Newtonian fluid is achieved. In this study, $m$ is fixed to $\eta_{0}$ while several quantities for $n$ are selected. This allows for the investigation of $n$ in the power law model during transient flows.

The other model is the Cross model, which has the following description:

$$
\frac{\eta-\eta_{\infty}}{\eta_{0}-\eta_{\infty}}=\frac{1}{1+k\left(\dot{\gamma}_{y x}\right)^{n}},
$$

where $n$ and $k$ are two fitting parameters whereas $\eta_{0}$ and $\eta_{\infty}$ are the limiting values of the apparent viscosity at low and high shear rates, respectively. In addition, for using shear rate and its independence from the coordinate system, Eq. (10) is applied [2].

$$
\begin{gathered}
\dot{\gamma}=\sqrt{-4 I I_{D}}, \\
D_{i j}=\frac{1}{2}\left(\frac{\partial u_{i}}{\partial x_{j}}+\frac{\partial u_{j}}{\partial x_{i}}\right),
\end{gathered}
$$

where $D_{i j}$ is the rate of deformation tensor, and $I I_{D}$ is the second invariant of $D_{i j}$. This representation of the shear rate in the $r, \theta, z$ coordinate system with assuming the one-direction flow pattern reduces to [2] and [24]:

$$
\dot{\gamma}=\sqrt{-4 I I_{D}}=\sqrt{-4 \times \frac{1}{2}\left((\operatorname{tr} \mathbf{D})^{2}-t r \mathbf{D}^{2}\right)}=\left|\frac{\partial v_{z}}{\partial r}\right| .
$$

\subsection{Initial Condition}

The fluid filled pipe is assumed to convey steady state flow before the transient event starts. So, the initial condition corresponds to the steady state flow. The momentum and continuity equations of the steady state flow can be written as:

$$
\begin{gathered}
\frac{\partial P}{\partial z}=\frac{-2 \tau_{0}}{R}, \\
\frac{\partial v_{z}}{\partial z}=0 .
\end{gathered}
$$

\subsection{Boundary Condition}

The transient flow in a reservoir-pipe-valve system is simulated. The quasi $2 \mathrm{D}$ analysis consists of three sets of boundaries including reservoir, valve, and internal pipe walls in contact with the flow. At the valve boundary, the velocity distribution is set to zero after the valve closure. A constant pressure head is associated with the reservoir boundary condition. The flow boundaries in contact with the pipe wall have zero velocity. These boundary equations can be written as follows:

$$
v_{z(r=R)}=0, \quad v_{z(\text { valve })}=0, \quad H_{\text {rezervoir }}=\text { const } .
$$

\section{NUMERICAL METHOD}

The proposed unsteady flow equations in the previous section are solved using the finite difference method. A fourth order Runge-Kutta scheme is used to integrate the system of equations in time. Spatial derivatives are discretized using second order central differencing scheme. Second order dissipative terms 
are added to eliminate numerical oscillations. These terms perform only in the high gradient region, and they are effectively switched off in smooth regions [7].

To start with the numerical implementation, the two Eqs. (4) and (6), are combined to one matrix-form equation with the unknown vector $\mathbf{W}=\left\{H V^{*}\right\}$ :

$$
\frac{\partial \mathbf{W}}{\partial t}+\mathbf{B} \frac{\partial \mathbf{W}}{\partial x}=\mathbf{C},
$$

where $\mathbf{B}$ and $\mathbf{C}$ are matrices of equation coefficients. The elements of vector $\mathbf{W}$ is sequentially evaluated from the discretized form of Eqs. (4) and (6) based on the Runge-Kutta scheme. The axial velocity profile is firstly evaluated from Eq. (6) so $V^{*}=V_{z}$. Then its average is applied in the continuity Eq. (4) to calculate pressure head $H$, thus herein $V^{*}=\bar{V}$. The dissipative terms represented by $\mathbf{A}(\mathbf{W})$ are added to Eq. (16) to suppress the artificial numerical fluctuations:

$$
\frac{\partial \mathbf{W}}{\partial t}+\mathbf{B} \frac{\partial \mathbf{W}}{\partial x}=\mathbf{C}+\mathbf{A}(\mathbf{W})
$$

The employed dissipative scheme is based on the Jamson method [27] which is directly added to the basic equations. It is evaluated as follows:

$$
\begin{gathered}
\mathbf{A}(\mathbf{W})=\frac{1}{\Delta t}\left[\mu_{i+1 / 2}\left(W_{i+1}-W_{i}\right)-\mu_{i-1 / 2}\left(W_{i}-W_{i-1}\right)\right], \\
\varepsilon_{i+1 / 2}=\frac{1}{2} \max \left(\alpha_{i+2}, \alpha_{i+1}, \alpha_{i}, \alpha_{i-1}\right),
\end{gathered}
$$

in which $\alpha$ is a numerical variable that behaves like a switch, to be on or off on high and low gradients of the unknowns, respectively. In this article, a total variation diminishing (TVD) scheme is adapted to distinguish high values of gradients according to:

$$
\begin{gathered}
\alpha_{i}=\frac{\left|W_{i+1}-2 W_{i}+W_{i-1}\right|}{(1-\omega) \Psi_{T V D}+\omega \Psi}, \\
\Psi=\left|W_{i+1}+2 W_{i}+W_{i-1}\right|, \\
\Psi_{T V D}=\left|W_{i+1}-W_{i}\right|+\left|W_{i}-W_{i-1}\right| .
\end{gathered}
$$

If $\omega$ is equal to zero, the above equation will be reduced to:

$$
\begin{gathered}
r=\frac{W_{i}-W_{i-1}}{W_{i+1}-W_{i}}, \\
\alpha_{i}=\frac{|1-r|}{1+1} .
\end{gathered}
$$

For calculating the average velocity of flow, the following equation is used, which is numerically computed by the Simpson integration scheme:

$$
V=\frac{\int_{0}^{R} 2 \pi r v_{z} d r}{\pi R^{2}} .
$$

\section{MODEL VERIFICATION}

In order to validate the mathematical model and corresponding numerical solution and its implementation, the computations are compared with experimental results. To this end, an experiment done by Holmboe and Rouleau [28] on a reservoirpipe-valve system with the following characteristics is considered. The pipe made of copper has an inner diameter of $0.025 \mathrm{~m}$ and a length of $36.09 \mathrm{~m}$. Pressure signals directly upstream of the valve and at the pipe midpoint are recorded. The operating fluid in the laminar flow condition (Reynolds number $=82$ ) is high-viscosity oil $\left(\mu=0.03484 \mathrm{~N} \cdot \mathrm{s} / \mathrm{m}^{2}\right)$, and the wave speed is measured to be $1324 \mathrm{~m} / \mathrm{s}$.

The test is initiated by sudden valve closure that causes excision of fluid flow in the valve place and creates oscillations in pressure and velocity propagating along the pipe.

Experiment results illustrate pressure values at various times after valve closure at two points along the pipe (valve and midpoint). In Figs. 1 and 2, non-dimensional experimental results taken from experiment [28] are compared with those of numerical results for the fluid pressure in the valve (Fig. 1) and midpoint (Fig. 2).

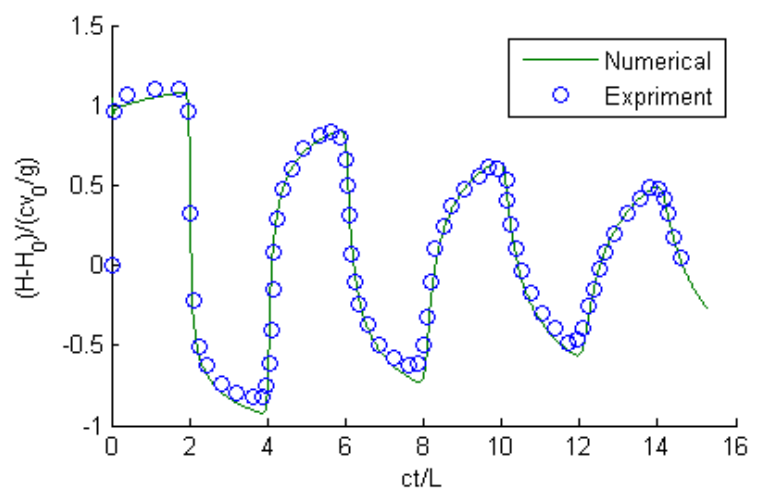

Fig. 1. Pressure time-history at the valve

According to these comparisons, there is good agreement between the numerical and experimental results, thus validating the proposed mathematical model and numerical implementation. 


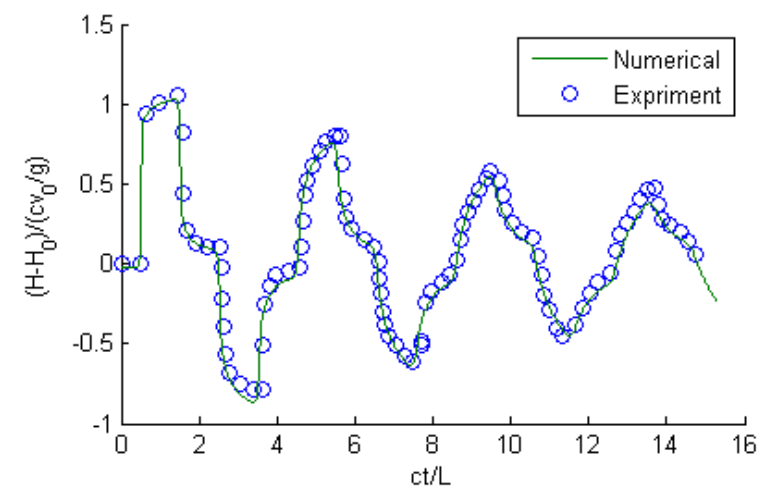

Fig. 2. Pressure time-history at midpoint of pipe

The average velocity of fluid flow (computed using Eq. (25)) at the reservoir and midpoint cross sections are provided in Fig. 3 and, as can be seen, the computational results have a similar pattern to those of the conventional one-dimensional solutions.

Another comparison is made for the nondimensional axial velocity profile at the midpoint at

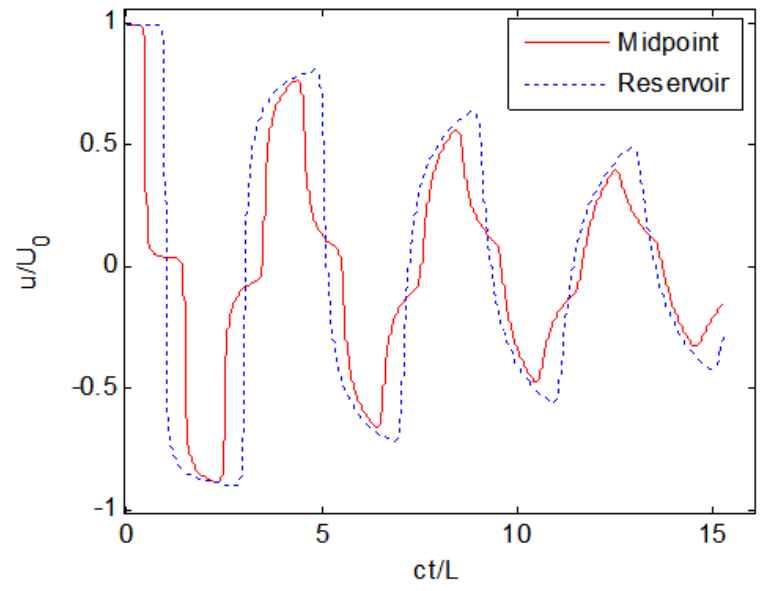

Fig. 3. Computational average velocity history at midpoint and reservoir

several time sections, being factors of the pipe length over the pressure wave speed $(L / c)$. The velocity profiles and their gradients can be compared with the corresponding computations provided by [7] (Fig. 4).
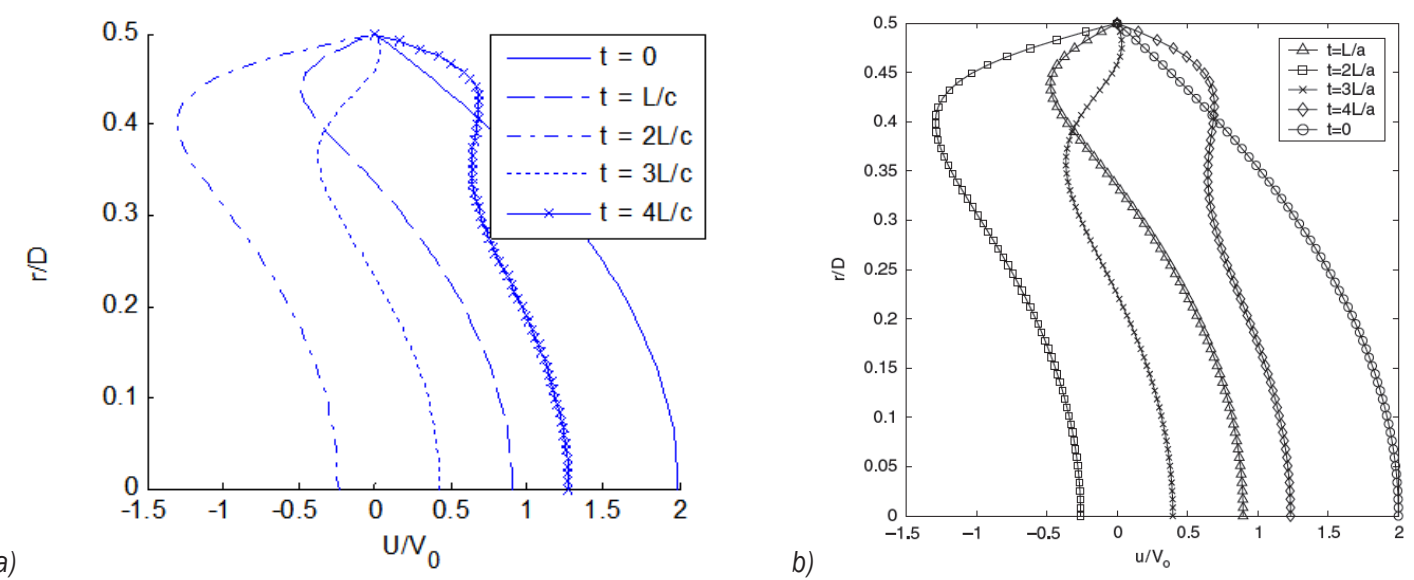

Fig. 4. Velocity profiles at the pipe midpoint for the laminar water hammer, a) present study, and b) Wahba [7])
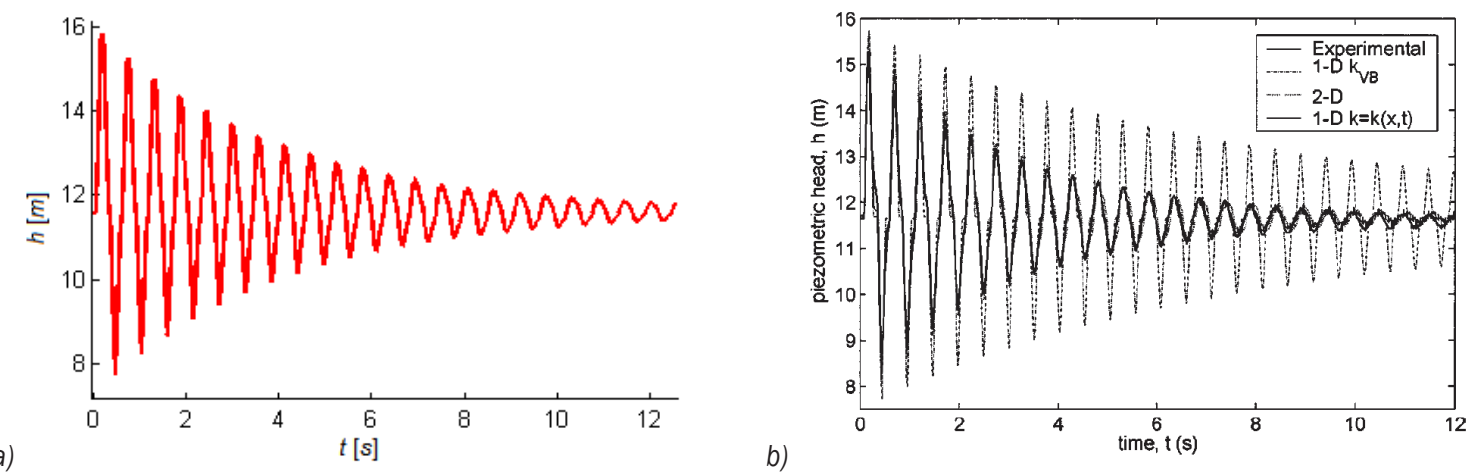

Fig. 5. Pressure time history at the pipe midpoint for the laminar water hammer, a) the calculations of the present study, and b) Brunone et al. study [20] 
Again, the consistency between the two set of results verifies the implemented computer code.

As more evidence for the correctness of the numerical model and its implementation, the results of Brunone et al. [20] were used. It explains that an experiment consists of a reservoir-copper pipe-valve system with $141.07 \mathrm{~m}$ length and $0.020 \mathrm{~m}$ inner diameter. The other specifications of the system are: Reynolds number is 815 , pressure wave speed 1120 $\mathrm{m} / \mathrm{s}$, valve closure $0.11 \mathrm{~s}$, and water temperature $17^{\circ} \mathrm{C}$. The modelling results of this study using the current simulation are compared with those of Brunone et al. [20] in Fig. 5. Fig 5b corresponds to the pressure heads obtained using a 1D model with unsteady friction [29] to [31] while Fig 5a is computed using the current $2 \mathrm{D}$ model.

\section{INVESTIGATION OF NON-NEWTONIAN FLUID EFFECTS}

To recognise the significance of the non-Newtonian fluid behaviour that is manifested in viscosity variations during a transient flow, a couple of numerical examples are presented and discussed in detail via several figures.

A pseudo plastic liquid that behaves as a shear thinning fluid is studied because it is the most common non-Newtonian fluid in applications.

The coefficients of the power law model are chosen to be $\left(\eta=\eta_{0}\right)$ and $n=0.8$ and 0.6. In the cross model the initial viscosity $\left(\eta_{0}\right)$ is chosen to be equal to that of Newtonian fluid (defined in the previous section for the verifying case), and the ultimate viscosity $\left(\eta_{\infty}\right)$ equates to $20 \%$ or $50 \%$ of the initial viscosity. The two remaining parameters of this model are assumed to be $n=2 / 3$ and $k=2$. Note that this way of allocation of the initial viscosity in the two models enables the computational results to be favourably compared with the corresponding simulations for the Newtonian fluids so as to discriminate the deviations introduced to the flow characteristics as a result of the nonlinear fluid property. The set above of coefficients for the power and Cross models leads the viscosity values to those presented in Figs. 6 and 7 for the various shear rates. As can be seen, the power law model (Fig. 7) considerably suffers from a lack of accuracy in the regions of low shear rate while the Cross model (Fig. 6) is in accordance with the reality that is herein assumed to be the Newtonian constant viscosity.

Several fluid properties defined via the power and Cross models are taken into account as the input fluid data for the transient flow analysis. The aim is to investigate transient pressures due to instantaneous downstream valve closure (see the previous section). Considering Joukowsky's pressure increase formula $\left(\Delta H=c V_{0} / g\right)$, the value of the transient pressure just after the excitation is directly related to the pressure wave speed and initial velocity (steady state).

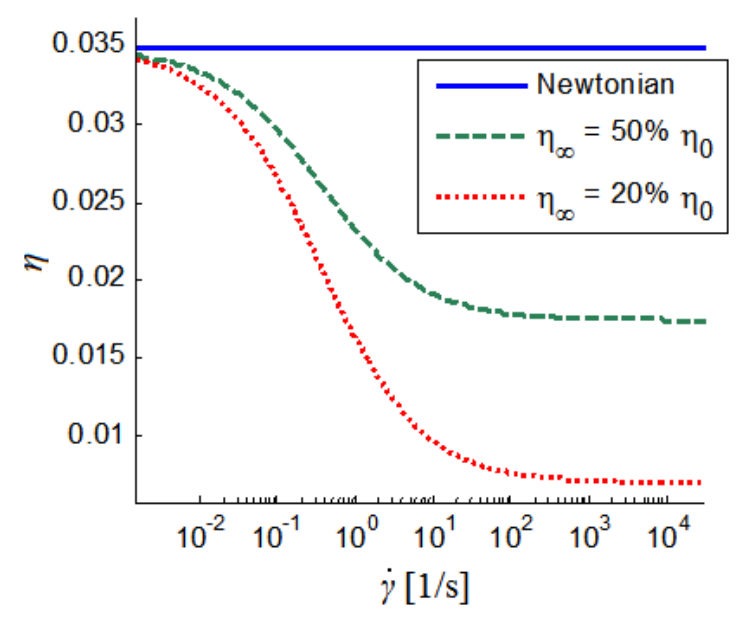

Fig. 6. Cross model viscosity variations vs. strain rate

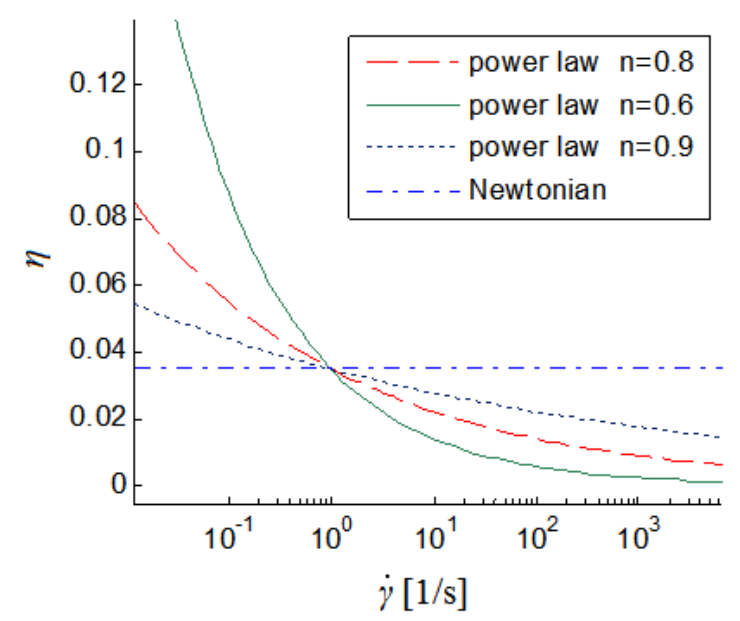

Fig. 7. Power law model viscosity variations vs. strain rate

These two quantities are kept unchanged so as to only scrutinize the non-Newtonian fluid properties during a transient flow. The defined various fluids, in turn, develop various pressure gradients and head loss during the steady state flow. The calculated head losses in the mentioned three power law cases are $2.693 \mathrm{~cm}, 1.343 \mathrm{~cm}$ and $0.6658 \mathrm{~cm}$ per metre and in the Cross model are $2.693 \mathrm{~cm}, 1.410 \mathrm{~cm}$ and 0.6404 $\mathrm{cm}$ per metre. According to the above conditions, the proposed numerical method produces the following pressures at the endpoint (valve) and the midpoint of pipe depicted in Figs. 8 and 9. Note that in all simulations, the flow pattern is laminar. 
The selected non-Newtonian fluids represent viscosities, though changing over the flow area but always smaller than that of the constant viscosity of Newtonian flow, (see Fig. 8 and Fig. 9). It means that smaller shear stresses develop on the pipe wall that correspond to less unsteady friction and causes to less pressure drop in the subsequent transient periods with respect to that of linear fluids. At the same time, during the first half period, the mentioned viscosity reduction of the shear thinning fluids enhances the fluid flow in the original direction and leads to less flow barrier and pressure gradient causing a smaller transient pressure rise in that time interval. In other words, a reduced packing effect is expected due to the reduced fluid viscosity, and this is in agreement with the computational figures that are provided for the pressure history at the valve and midpoint in Figs 8 and 9, respectively. The Figs $8 \mathrm{a}$ and $9 \mathrm{a}$ correspond to Cross and Figs $8 \mathrm{~b}$ and $9 \mathrm{~b}$ correspond to the power law model.

Another manifestation of non-Newtonian fluids is observed in velocity profiles at various time and space sections. This is shown for the aforementioned shear thinning fluids in Figs. 10 and 11. In these figures, the velocity profile in the middle section of the pipeline for the Newtonian and two non-Newtonian fluids are compared.

According to Figs. 10 and 11, the velocity distribution significantly changes as a result of the non-Newtonian fluid behaviour, and this change occurs throughout the unsteady fluid flow. The difference is such that with a reduction of fluid viscosity fluctuations, the variations of the velocity profile increase. In other words, the amplitude of the velocity gradient is increased in the flow crosssection. The growth in the velocity gradient in the vicinity of the pipe wall causes a drop in the viscosity value (shear thinning); in turn, this affects the values of fluid velocity and shear stress beside the pipe walls. Furthermore, the viscosity drop causes the maximum relative velocity to occur closer to the pipe walls. The velocity profiles also reveal that the central core area of flow has almost a rigid movement, and it is gradually affected by wall shear stress and viscosity variations of fluid near the pipe wall. In other words, the high values of wall shear stresses tend to penetrate in the core region and this pattern seems to be more progressive with the increase in viscosity variation
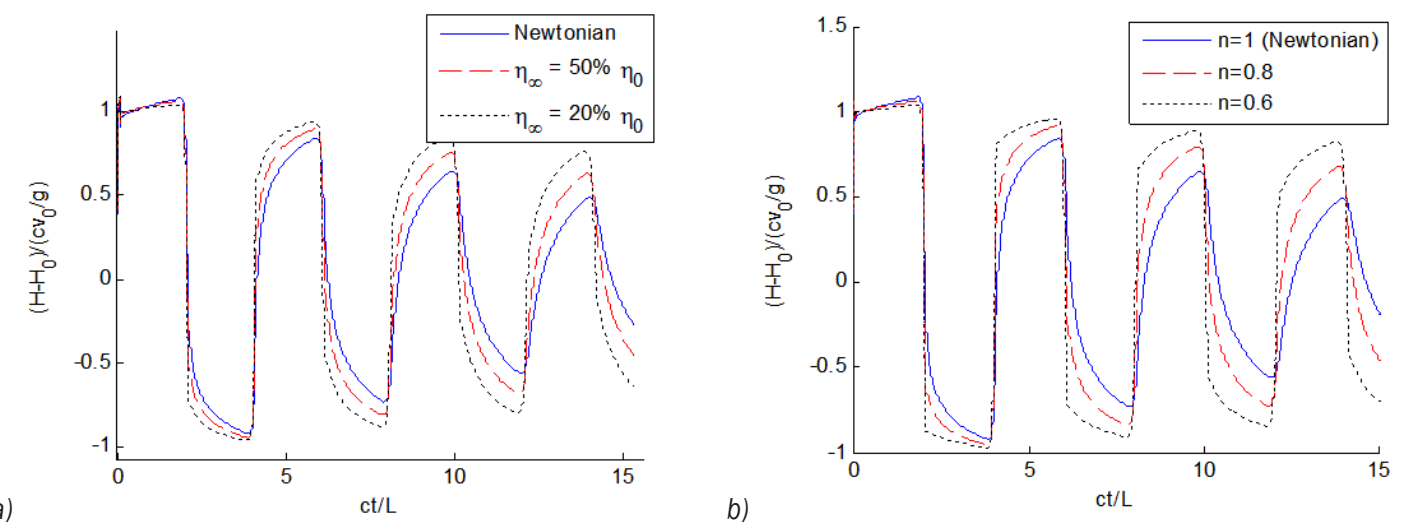

Fig. 8. Pressure time-history at the valve; a) Cross models, and b) power law
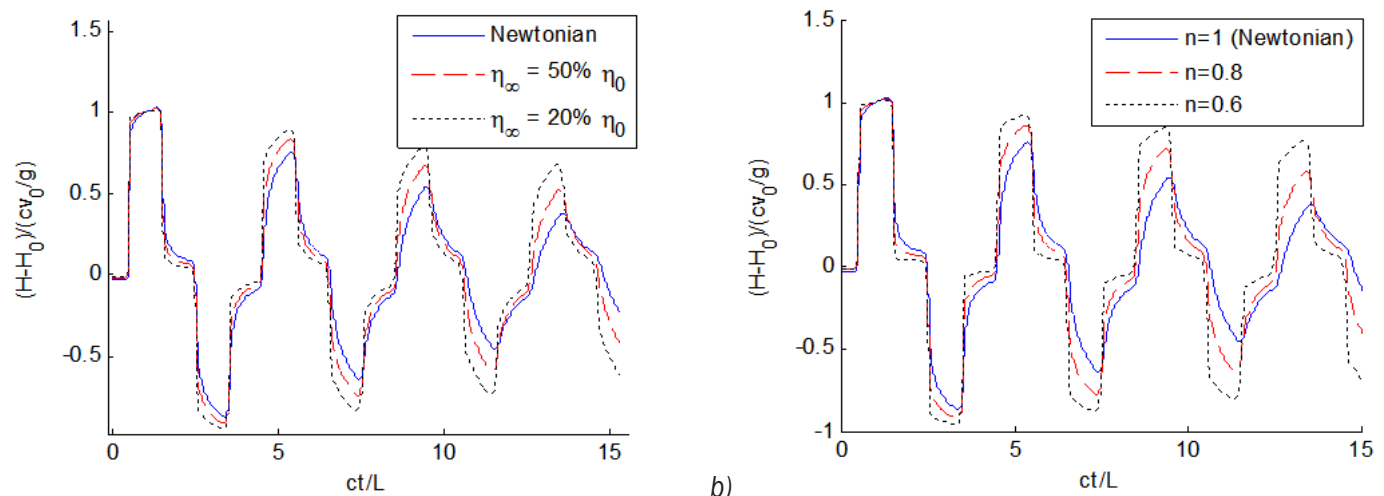

Fig. 9. Pressure time-history at the midpoint; a) Cross models, and b) right power law 

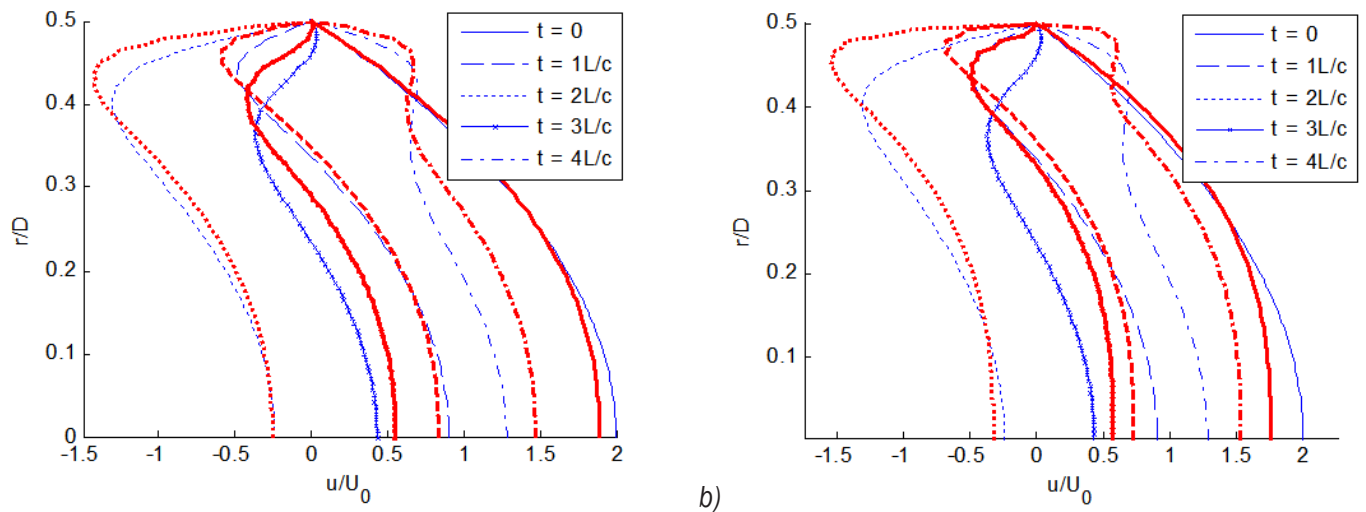

Fig. 10. Velocity profiles at the midpoint for the power law fluid model (thick line) vs. Newtonian model (thin line); a) $n=0.6$, and $b$ ) $n=0.8$
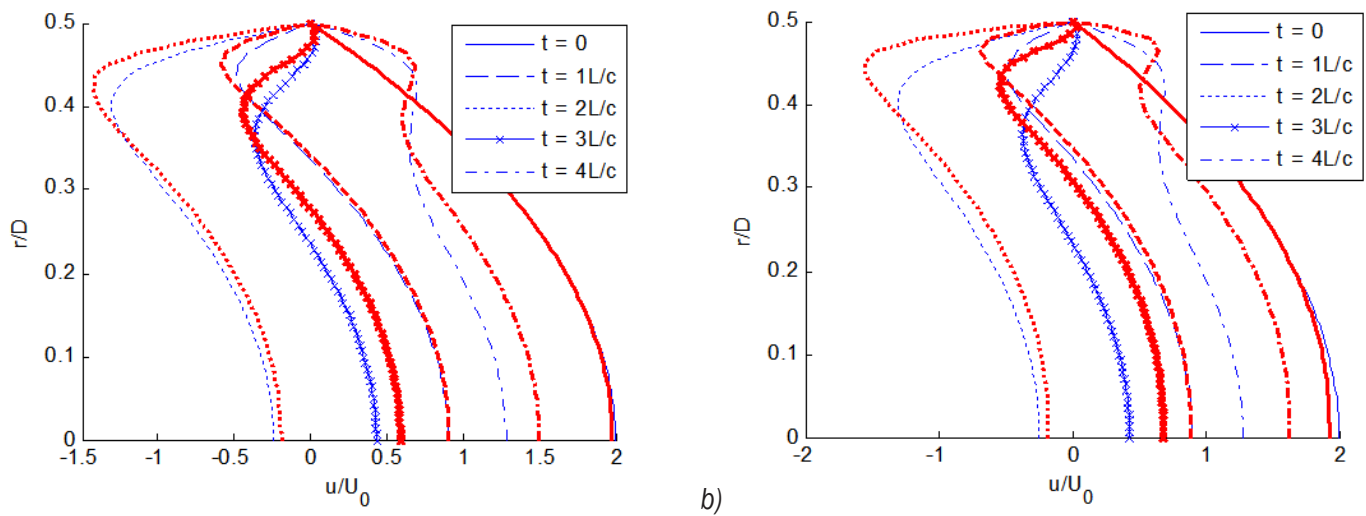

Fig. 11. Velocity profiles at the midpoint for the Cross fluid model (thick line) vs. Newtonian model (thin line);

a) $\eta_{\infty}=50 \% \eta_{0}$, and b) $\eta_{\infty}=20 \% \eta_{0}$

(with respect to the Newtonian fluid viscosity) in the present shear thinning fluids. This issue is explained in more detail by shear stress and viscosity distributions to be provided in the coming figures.

According to the depicted shear stress and viscosity profiles (Figs. 12 to 16 ), their values in the core area of the pipe cross section remain almost unchanged. In fact, the flow in this region demonstrates a rigid movement (no relative displacement).

According to the shear stress profiles at the pipe midpoint, the greater the shear thinning behaviour of the fluid, the less the expansion of the wall shear stresses to the core region of flow. Indeed, the area of rigid flow in the pipe cross section is extended, and the wall effects are more limited to the radial flow boundaries. In the meantime, the behaviours of the different non-Newtonian fluids in the core area of flow are remarkably similar to each other.

The fluctuations of viscosity in Figs. 15 and 16 during the fluid hammer can be interpreted in terms of velocity profiles in Figs 10 and 11 and the viscosity variations versus the strain rate in Figs.
6 and 7. According to Figs. 6 and 7, the maximum value of the shear rate corresponds to the least value of viscosity, and this occurs at the pipe wall annulus. This can be found from the first derivative of velocity profiles with respect to the pipe radius. In contrast, the minimum value of the shear rate leads to the largest values of viscosity, which in the power law model

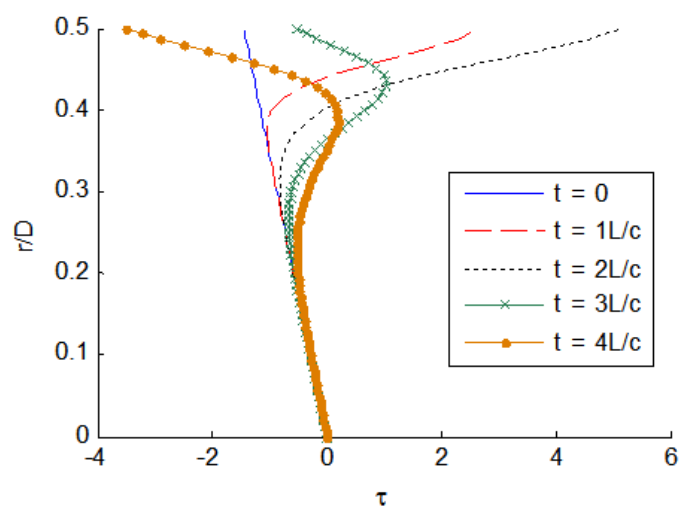

Fig. 12. Shear stress distribution for Newtonian model 

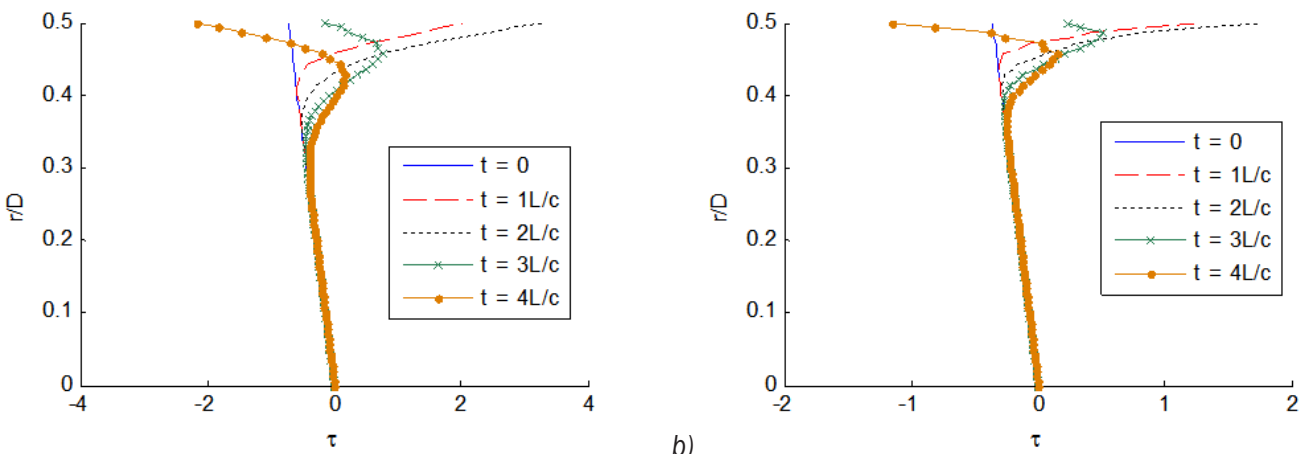

Fig. 13. Shear stress distribution for power law model; a) $n=0.8, b) n=0.6$

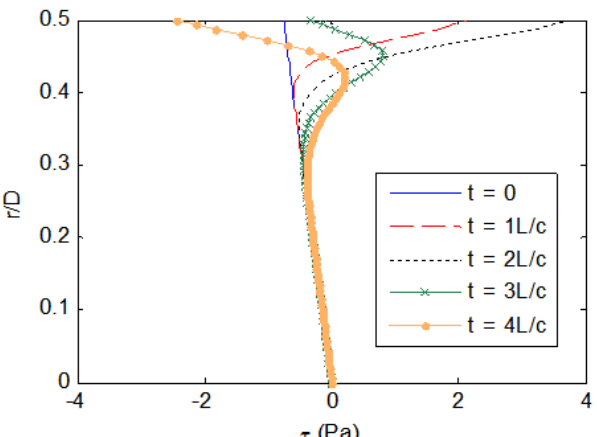

Fig. 14. Shear stress distribution for Cross model; a) $\eta_{\infty}=50 \% \eta_{0}$, and b) $\eta_{\infty}=20 \% \eta_{0}$

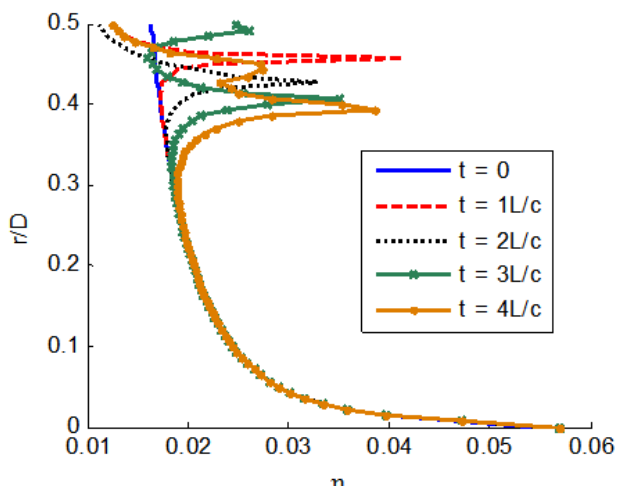

a)

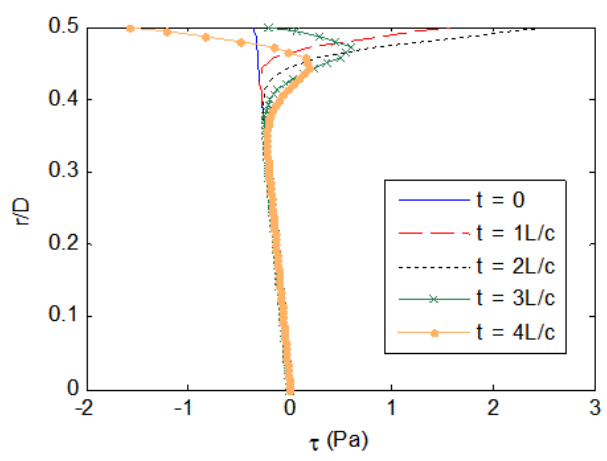

b)

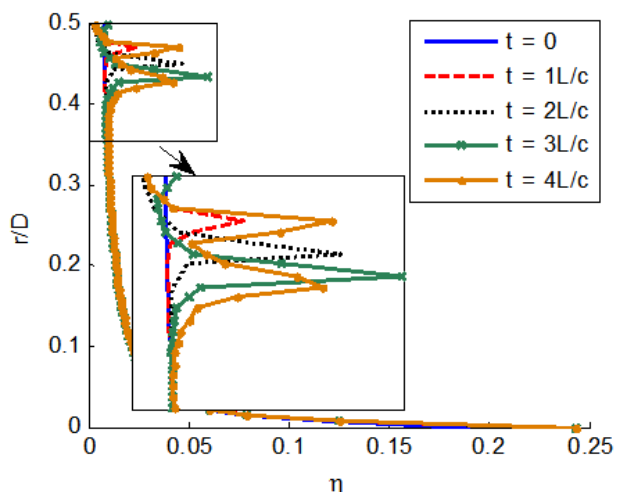

Fig. 15. Viscosity distribution in the pipe section for power law model; a) $n=0.8, b) n=0.6$

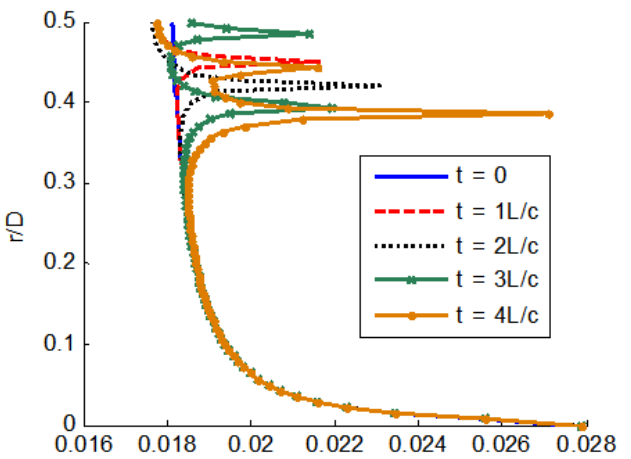

$\eta$

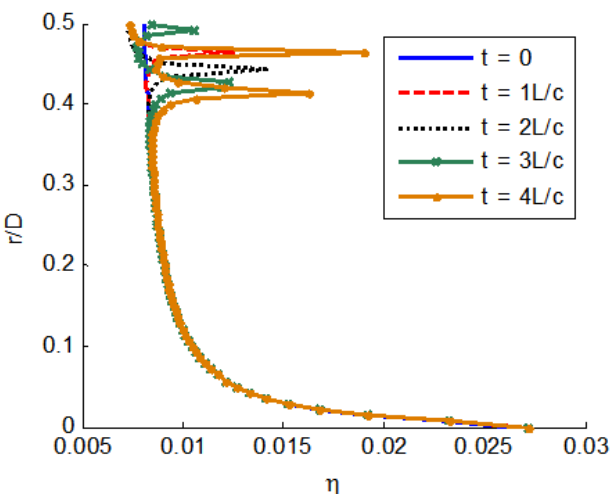

a)

Fig. 16. Viscosity distribution in the pipe section for Cross model; a) $\eta_{\infty}=50 \% \eta_{0}$, and b) $\eta_{\infty}=20 \% \eta_{0}$ 
tends to infinity and in the Cross model is a constant quantity called initial viscosity. According to the velocity profiles, around the central axis of the flow, the shear rate is zero, and it smoothly increases. This trend can be observed in the viscosity distribution, where it shows its largest value. There are more local maxima of viscosity in these figures that correspond to peaks in the velocity distribution. As an example, one can observe in Fig. $16 \mathrm{~b}$ at $t=4 \mathrm{~L} / \mathrm{c}$, which its maxima corresponds to the points indicated by arrows in Fig. 11b.

The aforementioned figures of viscosity and shear stress can be used to interpret the pressure time history results of non-Newtonian fluids. In fact, the increase in the shear thinning property of a liquid corresponds to viscosity and shear stress variations in the annulus of the pipe cross-section that are closer to the pipe walls. This trend, which is clearly manifested in Figs. 12 to 16, can also result from the velocity profiles in Figs. 10 and 11. This behaviour of the shear-thinning fluids indicates that the region of more energy dissipation is limited to a smaller area, which in turn leads to less energy loss and pressure drops during the transient event. This issue is demonstrated by Figs. 8 and 9, which show that the pressure history of the liquid with the greater shear-thinning property shows smaller pressure drop over time.

\section{CONCLUSION}

In this article, the non-Newtonian fluid effects in unsteady flows have been studied. Based on derived governing equations of transient non-Newtonian flows, a fourth-order Runge-Kutta numerical method has been used for the approximation of time phrases and second-order central difference scheme has been used for discretization in space. Furthermore, second-order dissipation phrases have been used for elimination of numerical fluctuations. In order to validate the proposed mathematical model and numerical solution, computational results have been compared with those of available experimental ones from the literature.

The differentiating pattern between Newtonian and non-Newtonian flows which mainly stems from the nonlinear dependency of fluid viscosity on velocity gradient is observed in pressure variation, velocity profile and wall shear stress. Non-Newtonian power law and Cross models verified previously were then investigated through case studies to see the axial velocity profile at various times. Some of the most important results follow.
The increase in the shear-thinning property of a liquid corresponds to the viscosity and shear stress variations in the annulus of the pipe cross-section that is closer to the pipe walls. This behaviour of the shearthinning fluids indicates that the region of greater energy dissipation is limited to a smaller area, which in turn leads to reduced energy loss and pressure drops during the transient event. The pressure history of the liquid with the greater shear-thinning property shows a reduced pressure drop over time.

Increasing the shear-thinning property of the nonNewtonian pseudoplastic fluid and, thus, the relative drop in the apparent viscosity decreased the amount of head loss in the pipe; comparatively, the pressure at the valve grows. Furthermore, due to the reduction of the apparent viscosity at the wall, a reduced linepacking effect is observed compared to Newtonian models.

The shear-thinning behaviour of non-Newtonian fluids causes the region of high gradient velocities to move towards the pipe walls and the maximum relative velocities occur closer to the radial boundaries, thus leading to severe fluctuations in the cross-sectional velocity profile.

The provided $2 \mathrm{D}$ computational results at a cross section reveal the significance of the non-slip boundary of the inner pipe wall during flow transients in terms of lags between the mean flow direction and velocities at several radii. The non-Newtonian fluid effect tends to vary the velocity profiles at each time.

In the midpoint of the pipe, a semi-rigid movement with negligible relative velocity variations was observed which is illustrated in terms of viscosity variations.

According to the velocity profiles, around the central axis of the flow, the shear rate is zero, and it smoothly increases. This trend can be observed in the viscosity distribution where it shows its largest value. There are more local maxima of viscosity in these figures that correspond to peaks in the velocity distribution.

\section{REFERENCES}

[1] Wylie, E.B., Streeter, V.L.A. Suo, L. (1993). Fluid Transients in Systems. Prentice Hall, Upper Saddle River.

[2] Bird, R.B., Armstrong, R.C. Hassager, O. (1987). Dynamics of Polymeric Liquids, 2nd ed., vol. 1. Wiley Interscience Publication, Hoboken.

[3] Chhabra, R.P., Richardson, J.F. (2011). Non-Newtonian Flow and Applied Rheology: Engineering Applications, $2^{\text {nd }}$ ed. Elsevier Science, Oxford. 
[4] Pinho, F.T. Whitelaw, J.H. (1990). Flow of non-Newtonian fluids in a pipe. Journal of Non-Newtonian Fluid Mechanics, vol. 34, no. 2. p. 129-144, DOI:10.1016/0377-0257(90)80015-R.

[5] Toms, B.A. (1948). Some observation on the flow of linear polymer solutions through straight tubes at large Reynolds numbers. Proceedings of 1 st International Congress on Rheology, vol. 2, p. 135-141.

[6] Ghidaoui, M.S., McInnis, D.A., Axworthy, D.H., Zhao, M. (2005). A review of water hammer theory and practice. Applied Mechanics Reviews, vol. 58, no. 1, p. 49-76, DOI:10.1115/1.1828050.

[7] Wahba, E.M. (2006). Runge-Kutta time-stepping schemes with TVD central differencing for the water hammer equations. International Journal for Numerical Methods in Fluids, vol. 52, no. 5, p. 571-590, D0l:10.1002/fld.1188.

[8] Riasi, A., Nourbakhsh, A., Raisee, M. (2009). Unsteady velocity profiles in laminar and turbulent water hammer flows. Journal of Fluids Engineering, vol. 131, no. 12, p. 121202, DOI:10.1115/1.4000557.

[9] Brunone, B. Berni, A. (2010). Wall Shear stress in transient turbulent pipe flow by local velocity measurement. Journal of Hydraulic Engineering, vol. 136, no. 10, p. 716-726, DOI:10.1061/(ASCE)HY.1943-7900.0000234.

[10] Brunone, B., Karney, B., Mecarelli, M., Ferrante, M. (2000). Velocity profiles and unsteady pipe friction in transient flow. Journal of Water Resources Planning and Management, vol. 126, no. 4, p. 236-244, Dol:10.1061/(ASCE)07339496(2000)126:4(236).

[11] Pezzinga, G. (1999). Quasi-2D Model for Unsteady Flow in Pipe Networks. Journal of Hydraulic Engineering, vol. 125, no. 7, p. 676-685, DOl:10.1061/(ASCE)0733-9429(1999)125:7(676).

[12] Pezzinga, G. (2000) Evaluation of Unsteady Flow Resistances by Quasi-2D or 1D Models. Journal of Hydraulic Engineering, vol. 126, no. 10. p. 778-785, Dol:10.1061/(ASCE)07339429(2000)126:10(778).

[13] Vardy, A.E., Brown, J.M.B. (2011). Laminar pipe flow with timedependent viscosity. Journal of Hydroinformatics, vol. 13, no. 4, p. 729-740, DOl:10.2166/hydro.2010.073.

[14] Wahba, E.M. (2013). Non-Newtonian fluid hammer in elastic circular pipes: Shear-thinning and shear-thickening effects. Journal of Non-Newtonian Fluid Mechanics, vol. 198, no. 0, p. 24-30, D0I:10.1016/J.jnnfm.2013.04.007.

[15] Keramat, A., Tijsseling, A.S., Q. Hou, Ahmadi, A. (2012). Fluidstructure interaction with pipe-wall viscoelasticity during water hammer. Journal of Fluids and Structures, vol. 28, p. 434455, DOI:10.1016/j.jfluidstructs.2011.11.001.

[16] Ahmadi, A., Keramat, A. (2010) Investigation of fluid-structure interaction with various types of junction coupling. Journal of Fluids and Structures, vol. 27, no. 7-8, p. 1123-1141, D0I:10.1016/j.jfluidstructs.2010.08.002.

[17] Soares, A.K., Covas, D.I.C., Carriço, N.J.G. (2012). Transient vaporous cavitation in viscoelastic pipes. Journal of Hydraulic Research, vol. 50, no. 2, p. 228-235, D0l:10.1080/00221686 .2012.669143.
[18] Hadj-Taïeb, L., Hadj-Taïeb E. (2009). Numerical simulation of transient flows in viscoelastic pipes with vapour cavitation. International Journal of Modelling and Simulation, vol 29, p. 206-213, DOl:10.2316/Journal.205.2009.2.205-5100.

[19] Pezzinga, G., Brunone, B., Cannizzaro, D., Ferrante, M., Meniconi, S., Berni, A. (2014). Two-Dimensional Features of Viscoelastic Models of Pipe Transients. Journal of Hydraulic Engineering, vol. 140, no. 8, p. 04014036, D0l:10.1061/ (ASCE)HY.1943-7900.0000891.

[20] Brunone, B., Ferrante, M., Cacciamani, M. (2005). Decay of Pressure and Energy Dissipation in Laminar Transient Flow. Journal of Fluids Engineering, vol. 126, no. 6, p. 7 , Dol:10.1115/1.1839926.

[21] Kim, S. (2011). Holistic Unsteady-Friction Model for Laminar Transient Flow in Pipeline Systems. Journal of Hydraulic Engineering, vol. 137, no. 12, p. 1649-1658, Dol:10.1061/ (ASCE)HY.1943-7900.0000471.

[22] Meniconi, S., Duan, H., Brunone, B., Ghidaoui, M., Lee, P., Ferrante, M. (2014). Further Developments in Rapidly Decelerating Turbulent Pipe Flow Modeling. Journal of Hydraulic Engineering, vol. 140, no. 7, p. 04014028 , DOI:10.1061/(ASCE)HY.1943-7900.0000880.

[23] Meniconi, S., Brunone, B., Ferrante, M., Massari, C. (2014). Energy dissipation and pressure decay during transients in viscoelastic pipes with an in-line valve. Journal of Fluids and Structures, vol. 45, no. 0, p. 235-249, Dol:10.1016/j. jfluidstructs.2013.12.013.

[24] Lai, W.M., Knovel, Krempl, E., Rubin, D. (2010). Introduction to Continuum Mechanics. $4^{\text {th }}$ ed., Butterworth-Heinemann, Elsevier, Amsterdam, Boston.

[25] Tijsseling, A.S. (1993). Fluid - Structure Interaction in Case of Waterhammer with Cavitation. PhD Thesis, Delft University of Technology, Delft.

[26] Tijsseling, A.S. (1996). Fluid-structure interaction in liquidfilled pipe systems: A review. Journal of Fluids and Structures, vol. 10, no. 2, p. 109-146, D0l:10.1006/jfls.1996.0009.

[27] Jameson, A., Schmidt, W., Turkel, E. (1981). Numerical Solutions of the Euler Equations by Finite Volume Methods Using Runge-Kutta Time-stepping Schemes, AIAA, Fluid and Plasma Dynamics Conference, Palo Alto.

[28] Holmboe, E.L., Rouleau, W.T. (1967). The Effect of Viscous Shear on Transients in Liquid Lines. Journal of Basic Engineering, vol. 89, no. 1, p. 174-180, DOl:10.1115/1.3609549.

[29] Zielke, W. (1968). Frequency-Dependent Friction in Transient Pipe Flow. Journal of Basic Engineering, vol. 90, no. 1, p. 109115, Dol:10.1115/1.3605049.

[30] Bratland, 0. (1986). Frequency-Dependent Friction and Radial Kinetic Energy Variation in Transient Pipe Flow. Proceedings of the $5^{\text {th }}$ International Conference on Pressure Surges, p. 95101.

[31] Vardy, A.E., Hwang, K. (1991). A characteristics model of transient friction in pipes. Journal of Hydraulic Research, vol. 29, no. 5, p. 669-684, Dol:10.1080/00221689109498983. 